U. Hoffarth ${ }^{1}$

R. Horch ${ }^{2}$

M. Steinert ${ }^{1}$

E. Stoelben ${ }^{1}$
Chirurgische Sanierung einer Aspergillomhöhle

\section{Surgical Treatment of Pulmonary Aspergilloma}

\section{Zusammenfassung}

Die Behandlung des Aspergilloms, sowohl die konservative als auch die operative, ist mit hohen Komplikationsraten verbunden. Die an einem Aspergillom erkrankten Patienten sind in der Regel nicht lungengesund. Der Therapieplan muss individuell, nach Allgemeinzustand, Lungenfunktion und Symptomatik, festgelegt werden. Mit der Darstellung dieser Kasuistik wird eine Möglichkeit der Plombierung einer Aspergillomhöhle durch einen gestielten, partiell deepithelialisierten Muskellappen als therapeutische Variante beschrieben. Der komplikationslose postoperative Verlauf und das exakte kosmetische Ergebnis haben dieses chirurgische Vorgehen berechtigt.

\section{Abstract}

Conservative as well as surgical treatment of pulmonary aspergilloma have a high complication rate. These patients usually have an underlying lung disease. The treatment needs to be adapted to the patient according to the general status, lungfunction and symptoms of the disease. This case report present a surgical treatment of an aspergillom. The aspergilloma cavity was filled with a vascularized pectoral muscle flap. The good cosmetic result without postoperative complications underline the efficiency of this procedure.

\section{Einleitung}

Das Aspergillom ist häufig eine Komplikation einer vorherbestehenden Lungenerkrankung [1,2]. Aus diesem Grund handelt es sich meist um Patienten mit eingeschränkter Lungenfunktion und reduziertem Allgemeinzustand. Die Resektion des befallenen Lungenanteils ist oft nicht möglich und die konservative Therapie nicht Erfolg bringend.
Bei unserem Fall handelt es sich um eine Patientin mit einer restriktiven Ventilationsstörung und einem erheblich gemindertem Allgemeinzustand (BMI von 12). Als besondere Komplikation kamen lebensbedrohliche Hämoptysen, unter konservativer Therapie hinzu, die bis zur Intubation geführt hatten. Aus diesen Gründen war ein limitierter chirurgischer Eingriff in Intubationsnarkose mit geringem Blutverlust unabdingbar. 


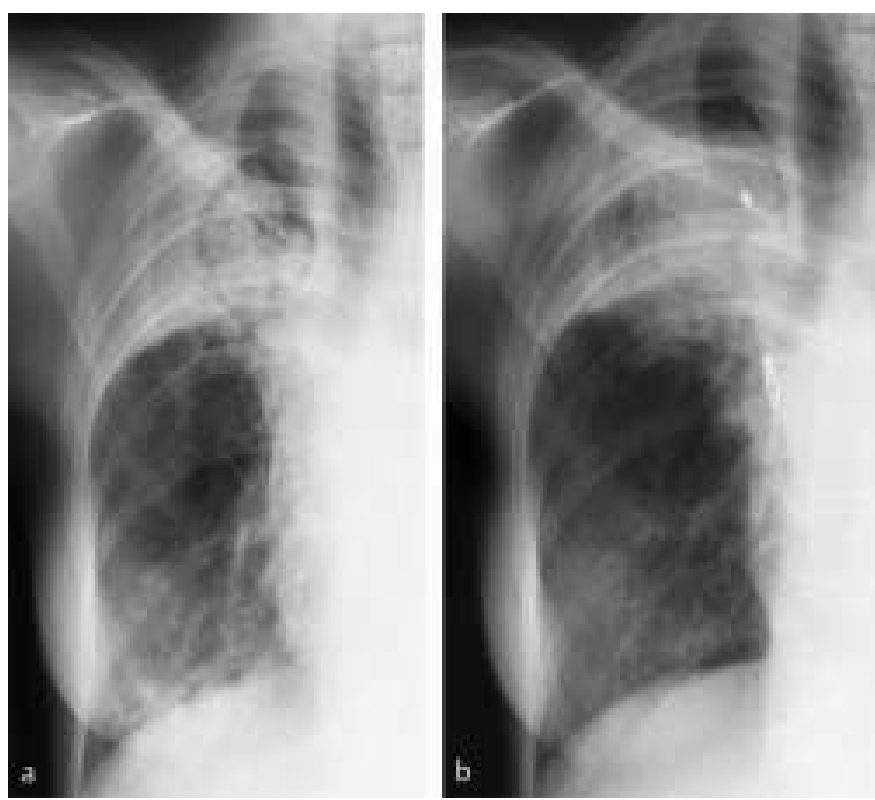

Abb. 1 a Präoperatives Röntgenbild (Mai 2000). b Präoperatives Röntgenbild nach Embolisierung (August 2002).

\section{Kasuistik}

Bei einer 1972 geborenen Patientin traten im Januar 1993 erstmals Schluckbeschwerden auf, des Weiteren bemerkte sie eine stetige Gewichtsabnahme. Als Ursache wurde eine Anorexia nervosa angenommen. Erst 18 Monate später, als die Patientin schon lange keine feste Nahrung mehr aufnehmen konnte, wurde erstmals eine Ösophagoskopie durchgeführt. Dabei wurde zunächst eine Ösophagusstenose eruiert, später konnte histologisch ein Ösophaguskarzinom gesichert werden.
Tab. 1 Anamnese

\begin{tabular}{|c|c|}
\hline 1993 & 21-jährige Patientin \\
\hline $1993-1994$ & $\begin{array}{l}\text { Diagnose Ösophaguskarzinom } \\
\text { Radio-Chemotherapie } \\
\text { Ösophagusresektion mit Magenhochzug }\end{array}$ \\
\hline 2000 & $\begin{array}{l}\text { Hämoptoe } \\
\text { Diagnose Aspergillom bds. re >li }\end{array}$ \\
\hline $2000-2002$ & $\begin{array}{l}\text { Rezidivierende Hämoptoe aus der rechten Lunge unter } \\
\text { Therapie } \\
\text { Progression d. Aspergilloms } \\
\text { Hämoptoe, Pneumonie, Beatmung }\end{array}$ \\
\hline Lungenfunktion & $\begin{array}{l}\text { FEV }_{1} 28 \% \text { der Norm } \\
\text { TLC } 95 \% \text { der Norm }\end{array}$ \\
\hline Gewicht & $35 \mathrm{~kg}$ \\
\hline Größe & $173 \mathrm{~cm}$ \\
\hline
\end{tabular}

Sie erhielt zunächst eine neoadjuvante Therapie. Danach erfolgte die transthorakale Ösophagektomie mit Magenhochzug. Als Nachbehandlung schloss sich eine mediastinale Bestrahlung und eine systemische Chemotherapie an. Die Rekonvaleszenz gestaltete sich komplikationslos.

Im Jahr 2000, also 7 Jahre nach Erstdiagnose des Ösophaguskarzinoms, trat eine akute Lungenblutung auf, deren Lokalisation im rechten Oberlappen war (Abb.1a). Die Patientin wurde beatmungspflichtig. In der bronchoalveolären Lavage konnte Aspergillus fumigatus im Trachealsekret nachgewiesen werden.

Eine chirurgische Therapie des Aspergilloms diskutierten wir bereits damals, entschieden uns aber wegen restriktiver Ventilationsstörung $\left(\mathrm{FEV}_{1}: 28 \%\right.$ der Norm) und schlechtem Allgemein-

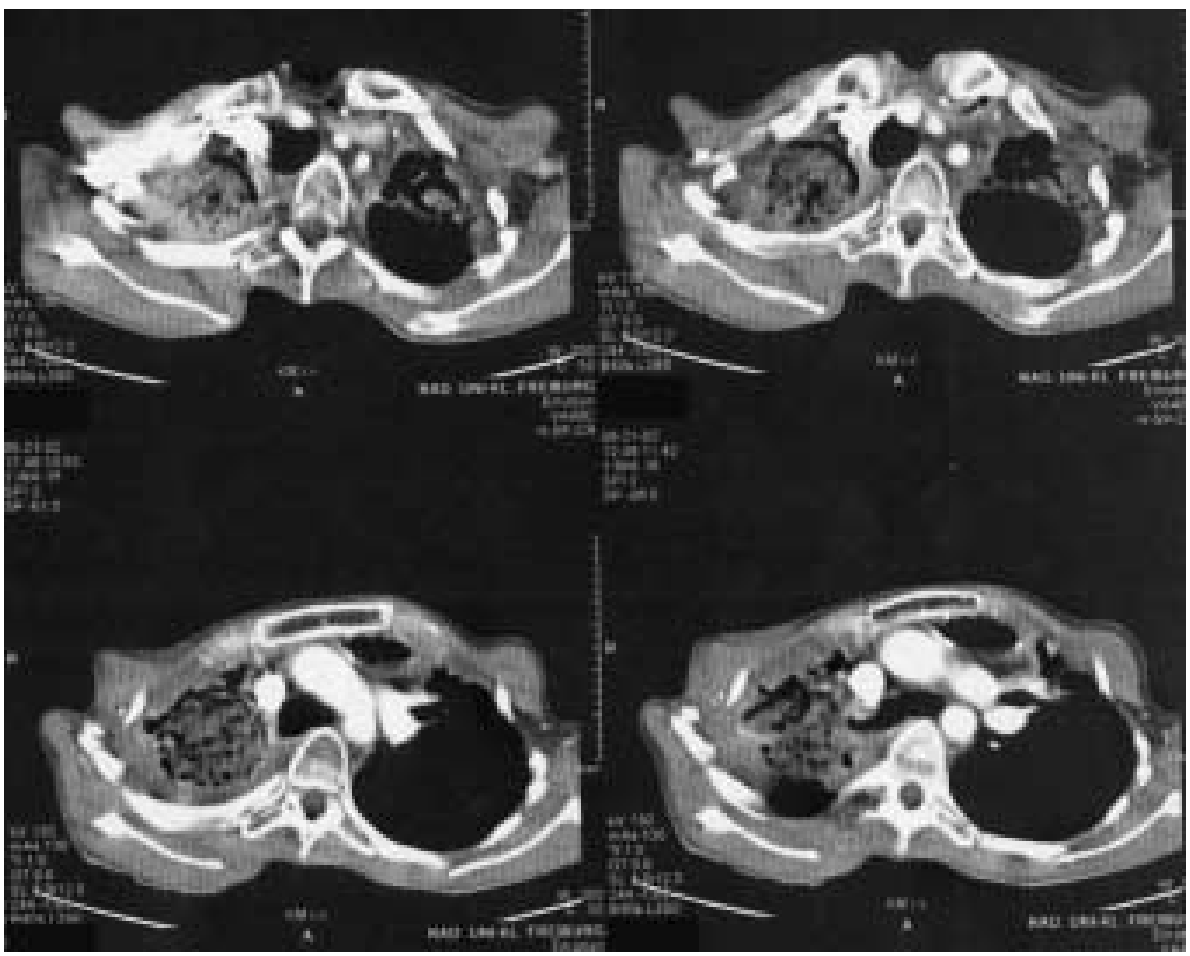

Abb. 2 Präoperatives CT-Thorax (August 2002). 


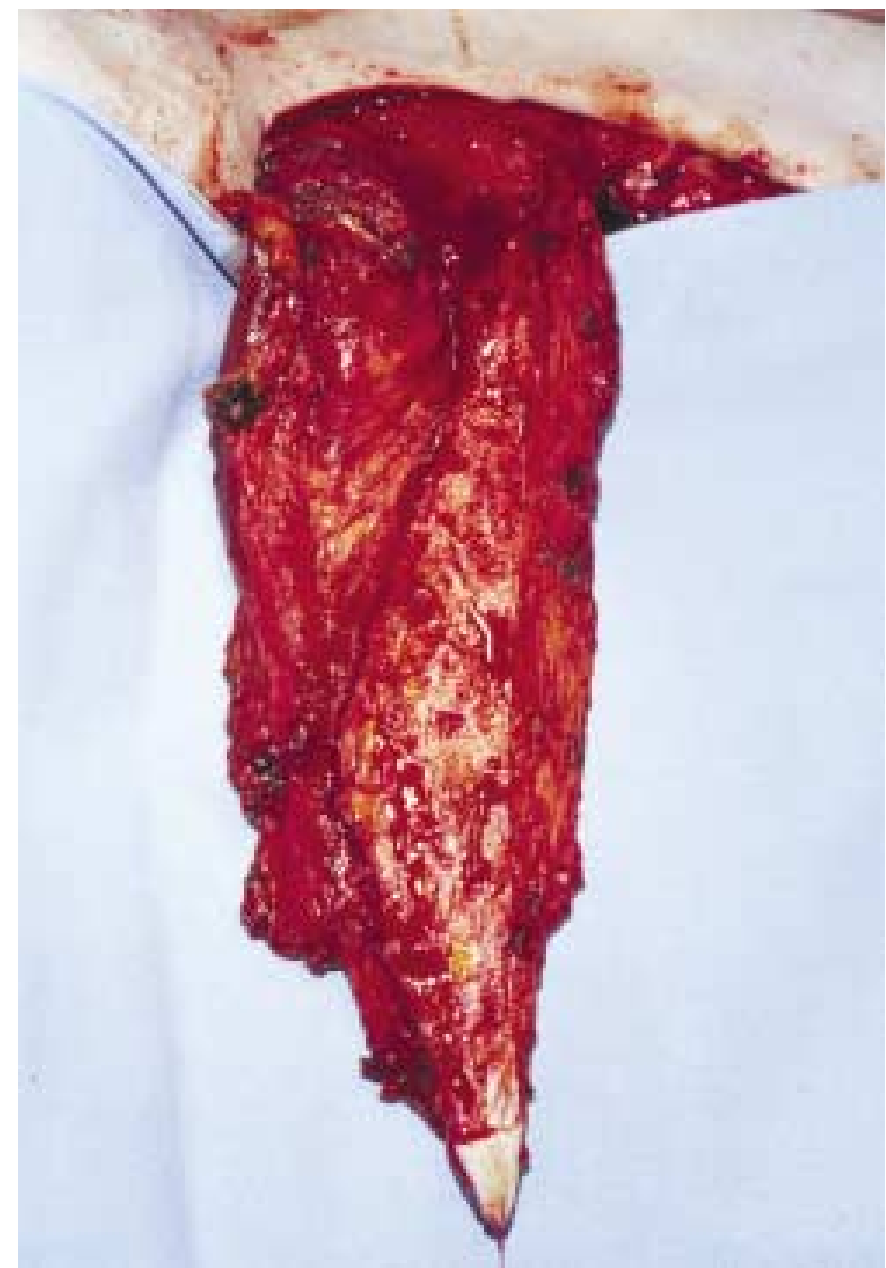

Abb. 3 Gestielter, voluminöser Haut-Muskellappen.

zustand (BMI 12) der Patientin für eine zunächst konservative Behandlung mit systemischer, antimykotischer Therapie, Embolisation einer Bronchialarterie sowie der Arteria mammaria interna und mit CT-gestützter Instillation von Amphotericin in die Höhle (Abb. 1b, 2). In einer Arbeit von Giron, wird diese perkutane Applikation als eine Erfolg bringende therapeutische Option beschrieben [3] (Tab.1).

Ende 2002 erfolgte abermals die stationäre Aufnahme wegen massiver Hämoptoe und wiederum Beatmungspflichtigkeit. Bei klinischem Progress der Erkrankung wurde die OP-Indikation Ende 2002 erneut kritisch diskutiert und aufgrund erfolgloser, konservativer Therapie gestellt.

Zunächst wurde ein an der A. acromioclavicularis gestielter, den M. pectoralis major enthaltender, Haut-Muskellappen von der ventralen Thoraxwand präpariert. Dieser wurde danach partiell deepithelialisiert (Abb. 3). Ein spindelförmiger Hautrest blieb erhalten. Über einen Zugang durch den 2. ICR sowie Teilresektion von zwei Rippen konnte die Höhle von ventral eröffnet werden. Intrathorakal war im Oberlappenbereich kein Lungenparenchym zu identifizieren. Die Höhle war etwa faustgroß, glattwandig und mit Granulationsgewebe durchsetzt, der Pilz stellte sich in gelblich-bröckliger Konsistenz dar (Abb.4). Am Boden der Aspergillomhöhle sah man den die Höhle drainierenden Bronchus. Der spindelförmige nicht deepithelialisierte Anteil des Muskellap-

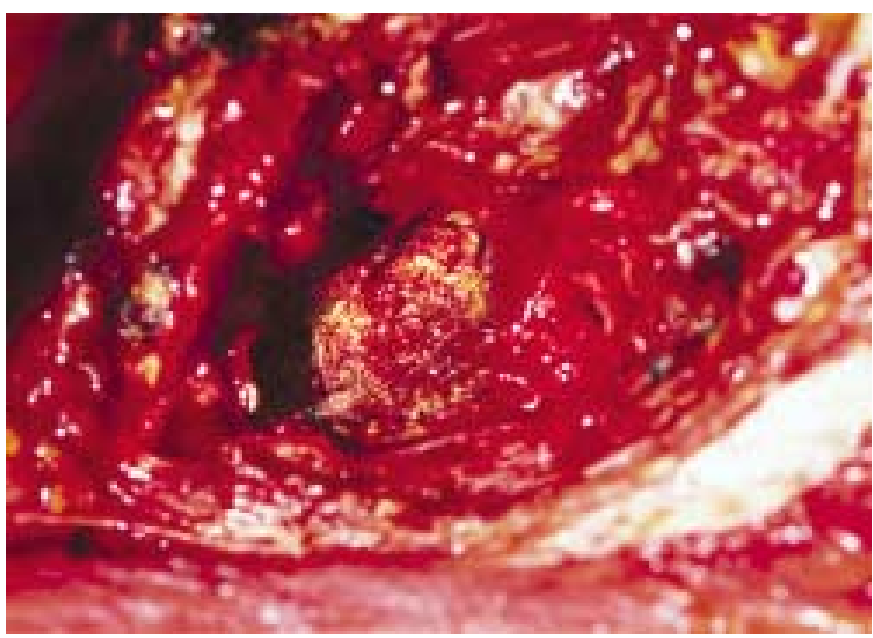

Abb. 4 Blick in die Aspergillomhöhle.

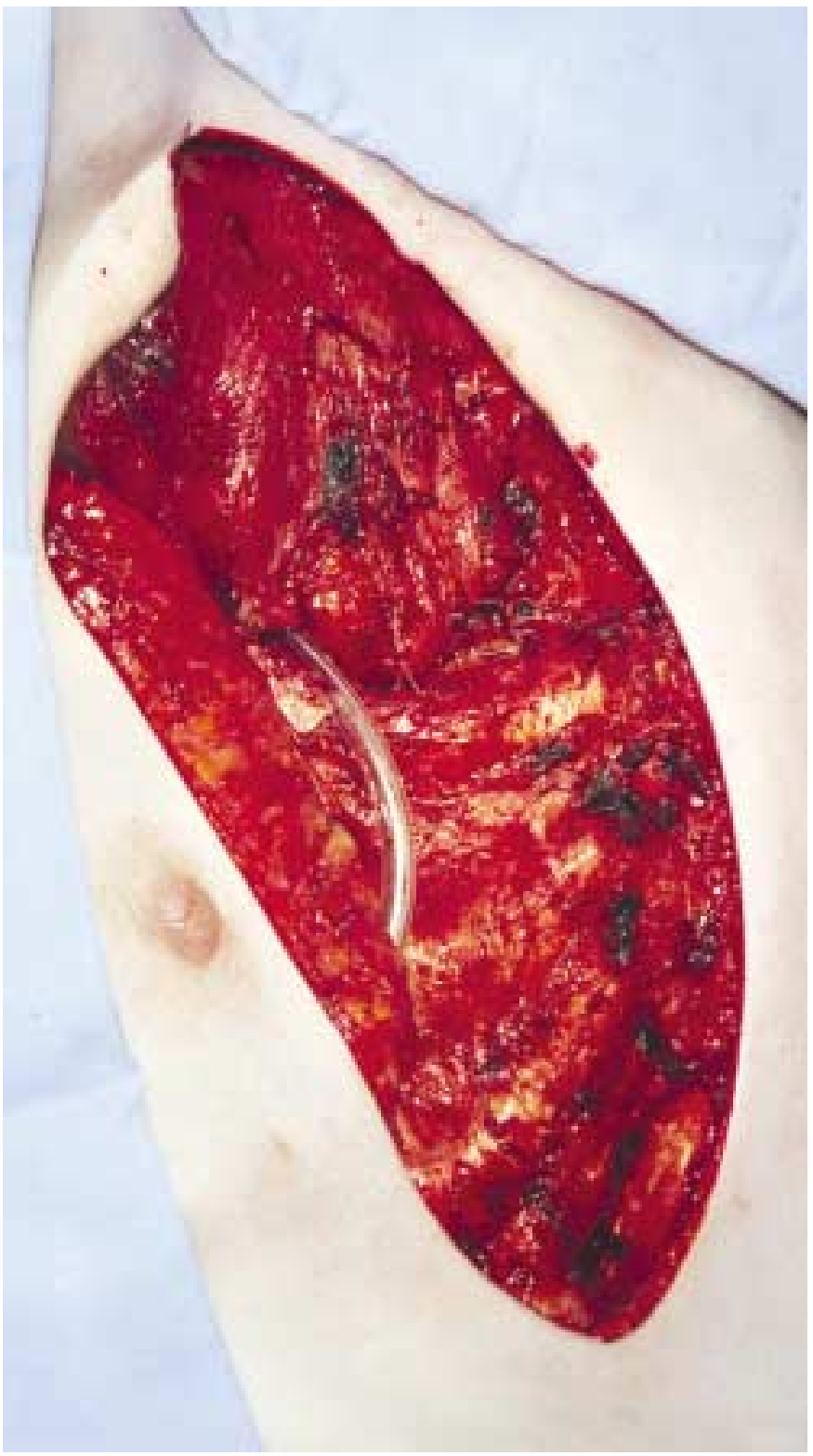

Abb. 5 Blick nach Schwenkung des Haut-Muskellappens nach intrathorakal 


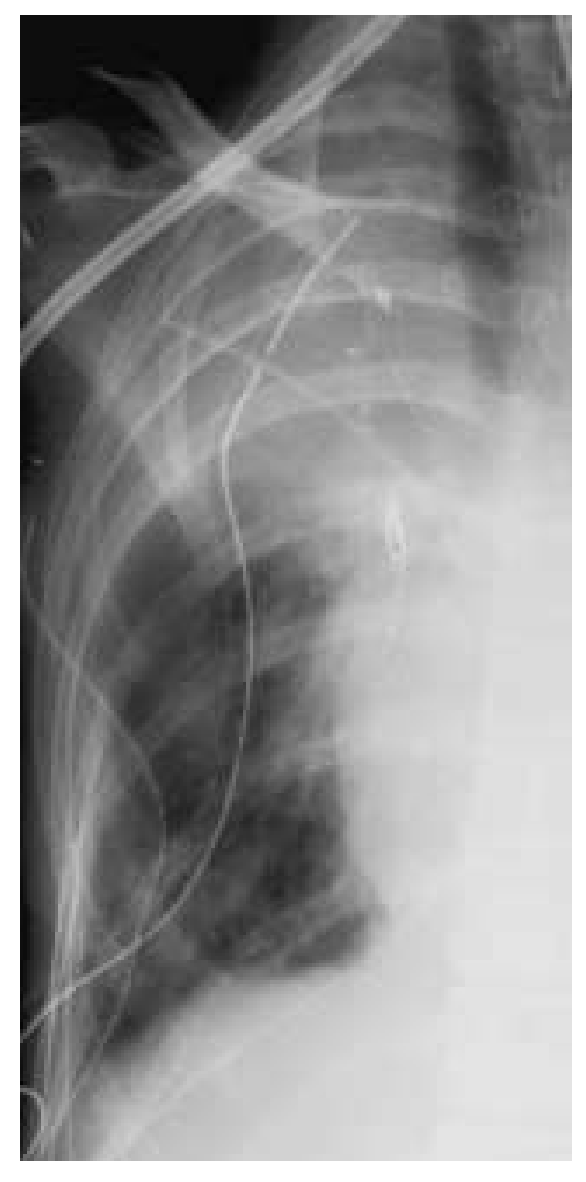

Abb. 6 Postoperatives Röntgenbild.

pens wurde auf den in die Kaverne reichenden Bronchus aufgenäht. Zuletzt konnte der gestielte Hautmuskellappen in die Höhle verlagert werden (Abb.5). Die Histopathologie zeigte im Brustwandresektat ein mäßig differenziertes Plattenepithelkarzinom. Der Kaverneninhalt ergab erwartungsgemäß ein Aspergillom. Der postoperative Verlauf war komplikationslos (Abb. 6). Die Patientin hat aktuell seit fast einem Jahr keine Hämoptoe mehr erlitten. Bezüglich des Ösophaguskarzinomrezidivs erfolgte eine onkologische Nachbetreuung.

\section{Diskussion}

Ein Aspergillom entsteht in einer präformierten Höhle und ist durch konservative Therapie nur schwer zugänglich. Die systemische Gabe von Amphotericin ist selten Erfolg bringend, da die Medikamente nicht in die Höhle penetrieren [2]. Giron teilt in seiner Studie mit 42 inoperablen Patienten gute Ergebnisse der CT-gestützten Instillation von Amphotericin mit [3]. Wie Soubani u. Mitarb. mitteilen, ist bei asymptomatischen Patienten keine Behandlung notwendig [2].

Engelmann u. Mitarb. empfehlen bei zwingendem Verdacht auf ein Aspergillom anhand von Klinik, radiomorphologischen Indizien und bei fehlenden Kontraindikationen ein operatives Vorgehen so zeitig wie möglich, bei Auftreten von Hämoptoe als zwin- gend notwendig [1]. Zur Vermeidung der Hämoptoe ist die Bronchialarterienembolisierung hilfreich, jedoch nur von zeitlich begrenztem Erfolg [2], wie dies auch in unserem Fall erkennbar ist.

Hinsichtlich der Operationstechniken stehen die Lobektomie bis zur Pneumonektomie $[1,2,4]$ zur Verfügung. Wie in unserem Fall berichtet, ist bei einem „destroyed lobe“ das Einbringen von vitalem Gewebe in die Aspergillomhöhle eine Alternative. Ziele waren es, den die Höhle drainierenden Bronchus aufzusuchen und zu verschließen, die Höhle zu debridieren und den „Pilzball“ zu entfernen.

In unserer Kasuistik waren der schlechte Allgemeinzustand (BMI 12) und eine restriktive Lungenfunktionsstörung $\left(\mathrm{FEV}_{1}: 28 \%\right.$ der Norm) maßgebend für die zunächst zurückhaltende, konservative Therapie. Eine nichtoperative Therapie sollte laut Engelmann u. Mitarb. nur bei OP-Verweigerung und Kontraindikationen, wie in unserem Fall, in Erwägung gezogen werden [1].

Da das Krankheitsbild unter Therapie erwartungsgemäß progredient war und die Patientin rezidivierend Hämoptoe erlitt, musste die OP-Indikation dringlich gestellt werden. Das Vorgehen mit Eröffnung der Höhle und Einbringung von vitalem Gewebe, reduzierte den introoperativen Blutverlust. Auch das Problem des postoperativen apikalen Pneumothorax bei mangelnder Ausdehnung der Lunge und dem Risiko des Aspergillusrezidivs wurde bei dieser Vorgehensweise entgegengewirkt. Diese Therapievariante hat sich im Fall unserer Patientin trotz eingeschränkter Lungenfunktion und schlechtem Allgemeinzustand komplikationslos durchführen lassen.

Das Tumorrezidiv war ein überraschender Befund. Ursprünglich war man davon ausgegangen, dass es sich bei der Aspergillombildung um eine Folge der Radio-Chemotherapie handelte.

Im Nachhinein muss man von einem zentral nekrotischen Tumorrezidiv mit Aspergillusbesiedelung ausgehen.

\section{Literatur}

${ }^{1}$ Engelmann C, Kracheel W, Krüger M. Chirurgische Therapie pleuropulmonaler Aspergillusinfektionen. Herz-Thorax-Gefäßchir 1999; 13: $126-132$

2 Soubani AO, Chandrasekar PH. The clinical spectrum of pulmonary aspergillosis. Chest 2002 Juni; 121 (6): 1988 - 1999

3 Giron J, Sans N, Poey C et al. Traitement percutané radiologique des aspergillomes pulmonaires inopérables. J Radiolog 1998; 79: 138-145

${ }^{4}$ Kaestel M, Meyer W, Mittelmeier HO et al. Pulmonary aspergillomaclinical findings and surgical treatment. Thorac Cardiovasc Surg 1999 Oct; 47 (5): 340-345

${ }^{5}$ Ono N, Sato K, Yokomise H et al. Surgical management of pulmonary aspergilloma. Role of single-stage cavernostomy with muscle transposition. Jpn J Thorac Cardiovasc Surg 2000; 48 (1): 56-59

${ }^{6}$ Regnard JF, Icard P, Nicolosi M et al. Aspergilloma: a series of 89 surgical cases. Ann Thorac Surg 1992; 54: 1159-1164

${ }^{7}$ Chen JC, Chang YL, Luh SP et al. Surgical treatment for pulmonary aspergillosis. Thorac Cardiovasc Surg 1969; 57: 642 -647 